\title{
AORTOGRAPHY IN CHILDREN WITH MYELOMENINGOCELE AND LUMBAR KYPHOSIS
}

\author{
B. FROMM, C. CARSTENS, F. U. NIETHARD, R. LANG \\ From the Orthopaedic University Hospital, Heidelberg
}

\begin{abstract}
In 21 children with myelomeningocele who underwent kyphectomy for congenital kyphosis of the lumbar spine, aortography revealed no case in which the aorta followed the spinal curvature. Many anomalies of the intercostal and segmental arteries were demonstrated which were only in part associated with deformities of the respective vertebral bodies. The kidneys, which were frequently malformed, often lay within the kyphosis and were therefore at risk of operative damage.

We conclude that the aorta is not at risk and that aortography is not usually necessary before kyphectomy, except in patients who have undergone prior abdominal surgery. Non-invasive methods (ultrasound, CT or MRI) should be used to detect malpositions and malformations of the kidneys.
\end{abstract}

The incidence of congenital kyphosis of the lumbar spine in children with myelomeningocele is reported to be between $8 \%$ and 15\% (Hoppenfeld 1967). Due to improvements in treatment the life expectancy of these patients has increased, and severe spinal deformity may develop and progress during the period of their growth. $\mathrm{Kyphosis}$ is commonest in the upper lumbar spine and may measure $80^{\circ}$ or more at the time of birth (Fig. 1) (Lindseth and Stelzer 1979). Without treatment, pressure sores, respiratory insufficiency, and intestinal problems may occur. Such patients cannot be fitted with orthotic devices and a surgical correction is often necessary at an early stage (Sharrard 1968).

The procedure, known as kyphectomy, includes the resection of the deformed segment of the spine, either in whole or in part, followed by arthrodesis. It is difficult to perform and prone to complications which may be fatal (Eckstein and Vora 1972). Aortography is usually performed pre-operatively to see whether the aorta follows the course of the kyphosis, and may therefore be injured when resecting the lumbar vertebrae. We have

B. Fromm, MD, Orthopaedic Surgeon

C. Carstens, MD, Assistant Professor

F. U. Niethard, MD, Professor

R. Lang, MD, Surgeon

Department of Paediatric Orthopaedics, Orthopaedic University Hospital, Schlierbacher Landstrasse 200a, 6900 Heidelberg, Germany.

Correspondence should be sent to Dr B. Fromm.

(C) 1992 British Editorial Society of Bone and Joint Surgery

$0301-620 \mathrm{X} / 92 / 5411 \$ 2.00$

J Bone Joint Surg [Br] 1992; 74-B :691-4. reviewed the aortograms of our patients to assess the likelihood of operative damage to the abdominal vessels.

\section{PATIENTS AND METHODS}

Between 1969 and 1990, 32 patients with myelomeningocele and kyphosis of the lumbar spine underwent kyphectomy at the Orthopaedic University Hospital of Heidelberg. We performed pre-operative aortography in 21 patients, seven male and 14 female. Their average age was five years eight months (one year eight months to 13 years two months) The average angle of the kyphosis was $118^{\circ}\left(72^{\circ}\right.$ to $\left.180^{\circ}\right)$. In 12 patients the apex of the kyphosis was at L2, in seven at L3 and in one each at L1 and L4.

Table I. Extent of the kyphosis in 21 patients

\begin{tabular}{ll}
\hline Level & Number \\
\hline T9 to L5 & 1 \\
T10 to L5 & 6 \\
T11 to L5 & 7 \\
T12 to L5 & 4 \\
L1 to L5 & 3 \\
\hline
\end{tabular}

The extent of the kyphosis was measured by the method of Cobb (1948). In all patients the whole of the lumbar spine was affected and in 18 the lower thoracic spine was also involved up to T9 (Table I). 


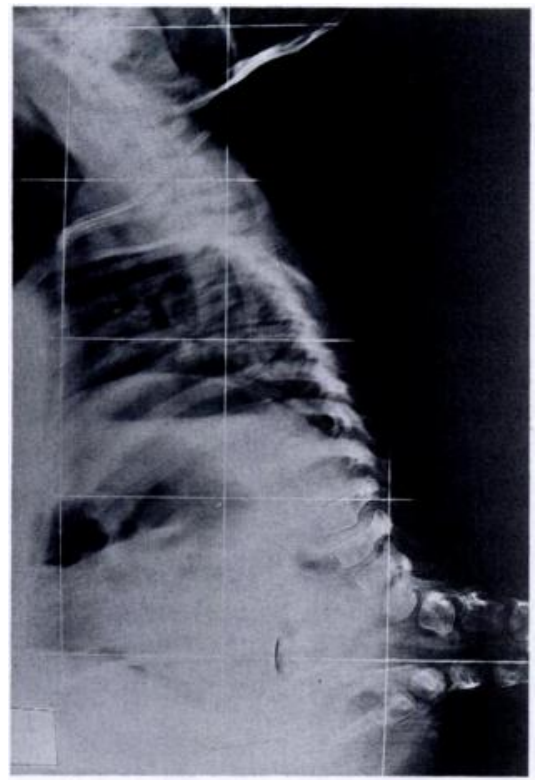

Fig. 1

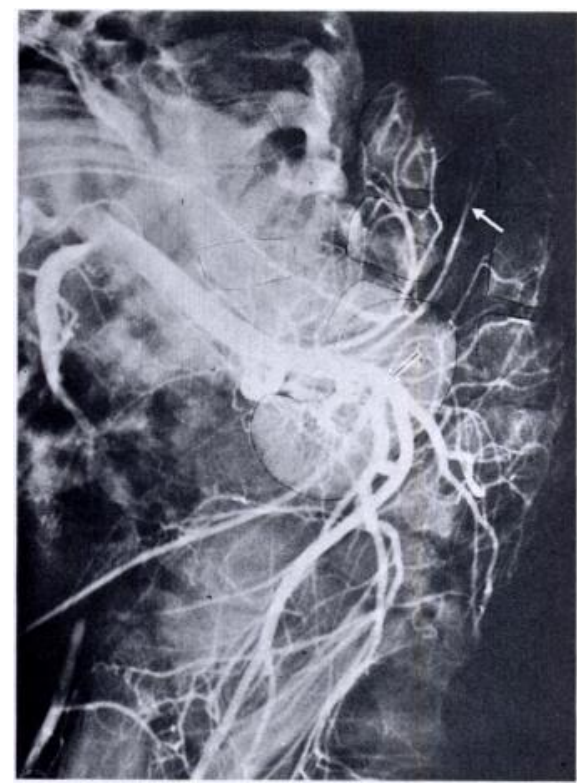

Fig. 2
Figure 1 - Lateral radiograph of a twoyear-old child in the sitting position, to show the $170^{\circ}$ kyphosis. Repeated pressure sores made lying on the back impossible. Figure 2 - Lateral angiogram of a severe $\mathrm{S}$ shaped kyphotic deformity shows $90^{\circ}$ kinking between the common iliac arteries and the abdominal aorta (open arrow). The single kidney, supplied by two renal arteries, is situated in front of the lowerend vertebra. Note the elongated lumbar arteries and their atypical segmentation (white arrow).
We performed aortography using a percutaneous Seldinger technique under general anaesthesia. The tip of the catheter was placed in the lower thoracic aorta. Films were taken in the anteroposterior and lateral projections and in some cases also in an oblique projection. The contrast medium in six cases was meglumine amidotrizoate $0.65 \mathrm{~g} / \mathrm{ml}$ (Angiografin, Schering $A G$, Berlin), and in 15 cases diatrizoate $0.1 \mathrm{~g} / \mathrm{ml}$ with meglumine amidotrizoate $0.66 \mathrm{~g} / \mathrm{ml}$ (Urografin, Schering AG, Berlin). About 10 to $20 \mathrm{ml}$ of contrast were injected with a flow rate between 5 and $10 \mathrm{ml}$ per second.

From the aortograms we recorded the course of the aorta, the origins of the coeliac axis and the superior and inferior mesenteric arteries, the number and course of the renal arteries, the level and position of the aortic bifurcation, the presence of aberrant segmental arteries, the calibre of the iliac vessels and of the abdominal aorta below the level of the mesenteric and renal branches, and the anatomical relationship of the kidneys to the apex of the kyphosis. The extent of the kyphosis and associated spinal deformities were documented from anteroposterior and lateral radiographs. The angle and site of the kyphosis were determined from radiographs taken with the patient in a sitting position (Fig. 1).

\section{RESULTS}

Considerable difficulties were experienced in performing the arteriography due to flexion contracture of the hips, the small calibre of the femoral arteries and kinking between the common iliac arteries and the abdominal aorta which measured $48^{\circ}$ to $92^{\circ}$ (mean $74^{\circ}$ ). In three cases a formal cut-down to the femoral artery was necessary; in another it was not possible to pass the catheter beyond the kink between the iliac artery and the aorta and the contrast medium had to be injected against the current. The only complication was haematoma formation at the puncture site, seen in four cases.

In no case did the abdominal aorta lie close to the vertebrae at the kyphosis. In 18 patients the vessel appeared to be strung across the deformity like a string across a bow (Figs 2 to 4 ). In the other three cases the aorta was closer to the kyphosis, but was not in a position where it was likely to be injured during operation.

In 13 of 15 patients over three years old, the calibre of the abdominal aorta below the origin of the inferior mesenteric artery was abnormally small (Fig. 5). The change in calibre was gradual, but with tapering to a diameter less than that of the large visceral arteries. There were similar findings in three of the six younger patients.

The lumbar arteries, originating from the aorta, followed a lengthy horizontal course to the dorsum of the kyphosis (Figs 2 and 3) and in six patients there were anomalies of the intercostal and lumbar arteries. In one patient these vessels did not originate from the aorta in pairs, but came from a single vessel arising from the aorta distal to the renal arteries. This vessel ascended over five segments lying close to the vertebrae and supplying each segment with two intercostal arteries (Fig. 3). In two patients, three vertebral bodies in the middle of the kyphosis were supplied by a single vessel, originating from the aorta. In another three patients two vertebral bodies were supplied in this way (Figs 2 and 3).

The caudal poles of the kidneys were directed towards the midline in 17 patients, the longitudinal axes of the kidneys representing an angle which was open cranially. In 14 patients the kidneys lay directly in front 


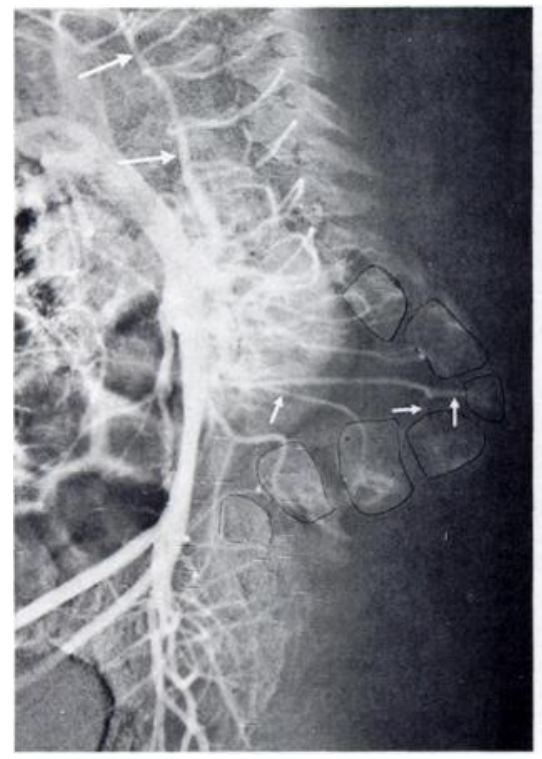

Fig. 3

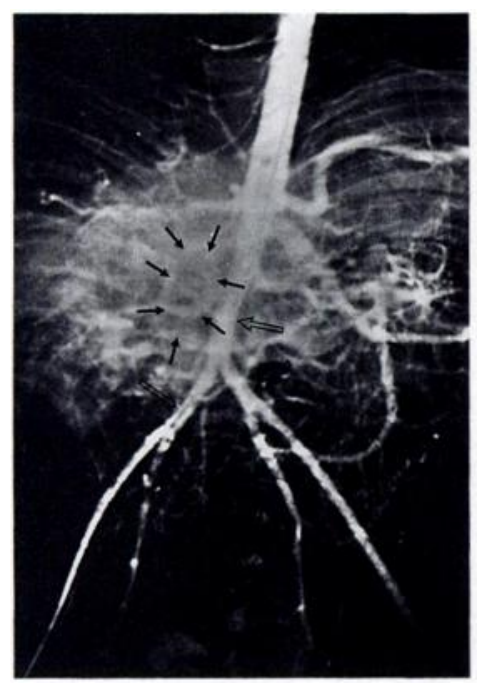

Fig. 5

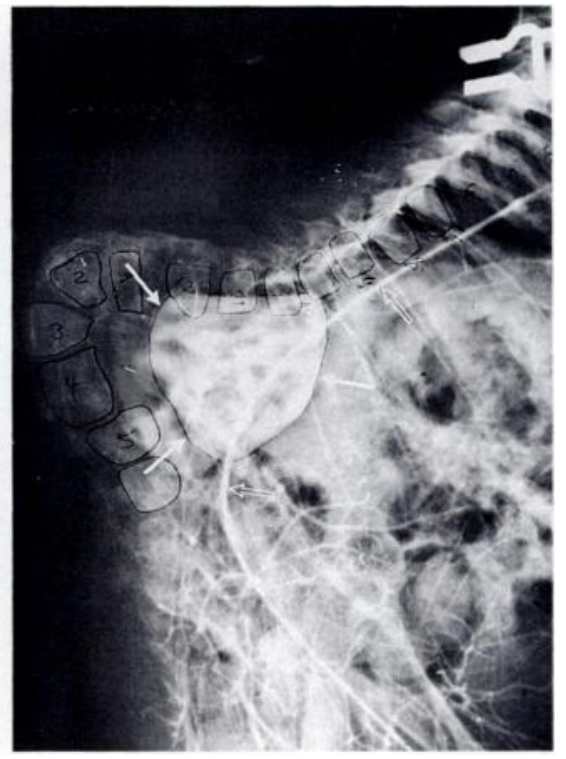

Fig. 4

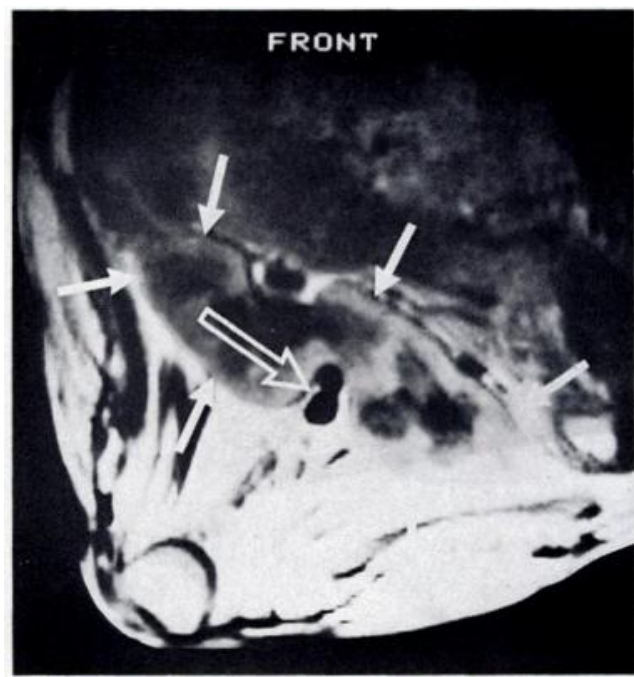

Fig. 6
Figure 3 - Lateral angiogram of a lumbar kyphotic deformity, bridged by the aorta. The kidneys lie in front of the upper-end vertebra of the kyphosis. Note the atypical course of the intercostal arteries (large arrows) and the elongated lumbar arteries with abnormal segmentation (smaller arrows). Figure 4 - Lateral view of a latephase angiogram with the catheter passing through the aorta (open arrows). The kidney lies within the kyphosis (white arrows).

Figure 5 - Anteroposterior angiogram. The single kidney is on the right (black arrows). The lower aorta and the iliac and femoral vessels (open arrows) are hypoplastic. Figure $6-C T$ of an angiographically suspected horseshoe kidney (white arrows). Note the distance between the abdominal aorta (open arrow) and the spine at the apex of the kyphosis.

of the proximal kyphotic segment (Fig. 3) and in six actually within the kyphosis (Fig. 4). In one patient they were situated ventral to the distal end-vertebra of the kyphosis (Fig. 2).

The frequency of renal anomalies revealed by the angiographic examination was surprisingly high. Five patients $(24 \%)$ had a solitary kidney (Figs 2 and 5), and a horseshoe kidney was suspected in another patient and confirmed by CT (Fig. 6). In some patients with a solitary kidney there was only one renal artery and in some there were two (Fig. 2).

\section{DISCUSSION}

We could find only three other articles on the abdominal aorta in children with myelomeningocele. These report a total of 15 patients (Hoppenfeld 1967; Watt and Park 1978; Loder et al 1991). Our results confirm those reported in the last two papers, in particular that the aorta usually follows a straight course in front of the kyphosis, like a bow-string. We found minimal retraction of the aorta into the kyphosis in only three patients. This makes it very unlikely that the vessel will be injured during kyphectomy.

The reported complications of kyphectomy have not been caused by injury to the aorta, but by an increase in intracranial pressure, or by meningitis after wound infection (Eckstein and Vora 1972). Loder et al (1991) reported one fatality due to complete occlusion of the aorta, but they consider that this was caused by retroperitoneal adhesions which developed after abdominal surgery performed before the kyphectomy. In our 
series of patients treated by kyphectomy, three died as a result of an increase in intracranial pressure. We had no instances of arterial occlusion or intra-operative arterial bleeding; we now believe that routine aortography before kyphectomy is not necessary. The procedure is technically difficult and the information gleaned does not justify the risks of additional anaesthesia in these children.

The exact localisation of the level of the aortic bifurcation was not possible, and we cannot comment, therefore, on the relative shortening of the aorta described by other authors. The abnormally small calibre of the aorta below the level of its mesenteric and visceral branches has been reported previously. The narrowing was more severe in the older patients in our series and we explain this as a result of the reduced requirement of blood supply to the paralysed legs, the vessels of which become hypoplastic with time.

Watt and Park (1978) and Loder et al (1991) found malformations of the lumbar arteries only at the apex of the kyphosis or below it. In our patients, malformations of the lumbar arteries were found both proximally and distally. Experimental studies on animals have shown that the vessels differentiate after vertebral segmentation (Amato and Bombelli 1959). Therefore, the prior malformation of the vertebral bodies in myelomeningocele could explain the presence of anomalies of the lumbar arteries, independent of the level of the apex of the kyphosis.

Several authors have described abnormal positions of the kidneys in these patients (Fernbach and Davis 1986; Loder et al 1991), but we are the first to report kidneys which lie completely within the kyphosis. There must be a risk of damaging the renal parenchyma during osteotomy of the proximal part of the kyphosis and, when the angle exceeds $120^{\circ}$, the distal osteotomy could lead to the same complication.

We were surprised to find so many malformations of the kidneys. The incidence of unilateral agenesis of the kidneys in children with myelomeningocele and congenital kyphosis is reported to be 1 in 500 to 1800 patients (Longo and Thompson 1952; Kelalis 1985). Watt and Park (1978) found only one case of horseshoe kidney in nine patients examined and Tori and Dickson (1980) reported a $21 \%$ incidence of all malformations of the urinary system in children with myelomeningocele and kyphosis. The reason for the combination of malformations seems to be the shared mesodermal origin of the kidneys and the vertebral bodies.

In summary, we think that aortography is usually unnecessary before kyphectomy, except in patients who have had previous urological or abdominal surgery. In these, the investigation may be useful to exclude retroperitoneal adhesions and to visualise anomalies of the retroperitoneal vessels. We do recommend preoperative examination for kidney malformations by

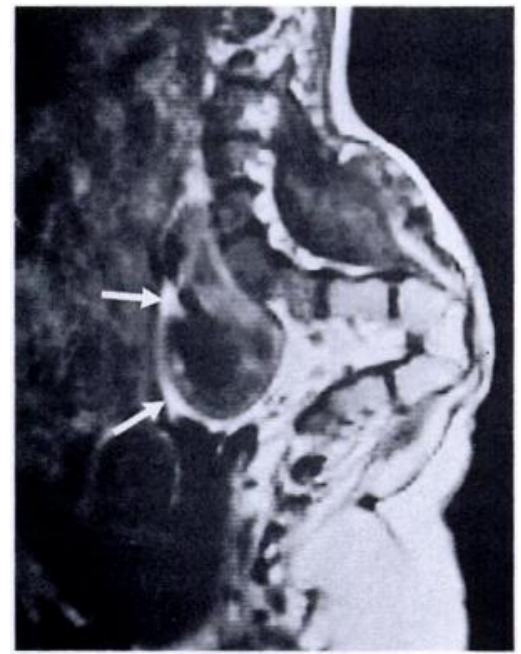

Fig. 7

MRI of a $150^{\circ}$ kyphosis to show the proximity of the kidney (arrows) to the upper end-vertebra.

non-invasive methods such as ultrasound, CT or MRI (Fig. 7).

No benefits in any form have been received or will be received from a commercial party related directly or indirectly to the subject of this article.

\section{REFERENCES}

Amato VP, Bombelli R. The normal vascular supply of the vertebral column in the growing rabbit. $J$ Bone Joint Surg [Br] 1959; 41B:782-95.

Cobb JR. Outline for the study of scoliosis. AAOS Instructional course lectures $1948 ; 5: 261-75$.

Eckstein HB, Vora RM. Spinal osteotomy for severe kyphosis in children with myelomeningocele. J Bone Joint Surg [Br] 1972; 54 B:328-33.

Fermbach SK, Davis TM. The abnormal renal axis in children with spina bifida and gibbus deformity: the pseudohorseshoe kidney. J Urol 1986; 136:1258-60

Hoppenfeld S. Congenital kyphosis in myelomeningocele. $J$ Bone Joint Surg [ Br] 1967; 49-B:276-80.

Kelalis PP. Anomalies of the urinary tract. In: Kelalis PP, King LR, Belman AB, eds. Clinical paediatric urology. Vol 2, Second edition. Philadelphia: WB Saunders, 1985 ; 643-54.

Lindseth RE, Stelzer L Jr. Vertebral excision for kyphosis in children with myelomeningocele. J Bone Joint Surg [Am] 1979; 61-A: 699-704.

Loder RT, Shapiro P, Towbin R, Aronson DD. Aortic anatomy in children with myelomeningocele and congenital lumbar kyphosis. $J$ Pediatr Orthop 1991; 11:31-5.

Longo VJ, Thompson GJ. Congenital solitary kidney. J Urol 1952; $68: 63-8$.

Sharrard WJW. Spinal osteotomy for congenital kyphosis in myelomeningocele. J Bone Joint Surg [Br] 1968; 50-B:466-71.

Tori JA, Dickson JH. Association of congenital anomalies of the spine and kidneys. Clin Orthop 1980; 148:259-62.

Watt I, Park WM. The abdominal aorta in spina bifida cystica. Clin Radiol 1978; $29: 63-8$. 